\title{
Burn depth determination using high- speed polarization-sensitive Mueller optical coherence tomography with continuous polarization modulation
}

\author{
Miloš Todorović, Jun Ai, David Pereda Cubian, George \\ Stoica, Lihong Wang
}

Miloš Todorović, Jun Ai, David Pereda Cubian, George Stoica, Lihong Wang, "Burn depth determination using high-speed polarization-sensitive Mueller optical coherence tomography with continuous polarization modulation," Proc. SPIE 6079, Coherence Domain Optical Methods and Optical Coherence Tomography in Biomedicine X, 60792B (20 February 2006); doi: 10.1117/12.649024

SPIE. Event: SPIE BiOS, 2006, San Jose, California, United States 


\title{
Burn depth determination using high-speed polarization-sensitive Mueller optical coherence tomography with continuous polarization modulation
}

\author{
Miloš Todorović* a, Jun Ai ${ }^{a}$, David Pereda Cubian ${ }^{\mathrm{a}}$, George Stoica ${ }^{\mathrm{b}}$, \\ Lihong Wang ${ }^{\mathrm{a}}$ \\ ${ }^{a}$ Optical Imaging Laboratory, Department of Biomedical Engineering, \\ Texas A\&M University, 3120 TAMU, College Station, TX 77843-3120 \\ ${ }^{\mathrm{b}}$ Department of Pathobiology, Texas A\&M University, 5547 TAMU, College Station, TX \\ 77843-5547
}

\begin{abstract}
National Health Interview Survey (NHIS) estimates more than 1.1 million burn injuries per year in the United States, with nearly 15,000 fatalities from wounds and related complications. An imaging modality capable of evaluating burn depths non-invasively is the polarization-sensitive optical coherence tomography. We report on the use of a high-speed, fiber-based Mueller-matrix OCT system with continuous source-polarization modulation for burn depth evaluation. The new system is capable of imaging at near video-quality frame rates ( 8 frames per second) with resolution of $10 \mu \mathrm{m}$ in biological tissue (index of refraction: 1.4) and sensitivity of $78 \mathrm{~dB}$. The sample arm optics is integrated in a hand-held probe simplifying the in vivo experiments. The applicability of the system for burn depth determination is demonstrated using biological samples of porcine tendon and porcine skin. The results show an improved imaging depth $(1 \mathrm{~mm}$ in tendon) and a clear localization of the thermally damaged region. The burnt area determined from OCT images compares well with the histology, thus proving the system's potential for burn depth determination.
\end{abstract}

Keywords: burn depth, polarization modulation, polarization, OCT, birefringence, phase retardation, Jones matrix, Mueller matrix.

\section{INTRODUCTION}

National Health Interview Survey (NHIS) estimates more than 1.1 million burn injuries per year in the United States. Approximately 45,000 of these injuries require hospitalization and roughly 4,500 people die. In addition, up to 10,000 people in the United States die every year of burn-related infections. ${ }^{1} \mathrm{~A}$ burn is defined as tissue damage caused by a variety of agents, such as heat, chemicals, electricity, sunlight, or nuclear radiation. The clinical significance of a burn depends on the depth of the burn and the area affected by it. ${ }^{2}$

Traditionally, burns were classified using a degree scale. Lately, a new nomenclature has gained recognition were burns are primarily classified according to their depth and the tissue layers involved. ${ }^{3}$ In superficial burns, the dermis is spared and often the epidermis, although devitalized, still exists. Superficial partial-thickness burns extend through the epidermis into the papillary layer of the dermis, while deep partial-thickness burns affect the reticular layer of the dermis. Finally, full-thickness burns extend through the epidermis and dermis into the subcutaneous

${ }^{*}$ milos@tamu.edu; http://oilab.tamu.edu

Coherence Domain Optical Methods and Optical Coherence Tomography in Biomedicine X

edited by Valery V. Tuchin, Joseph A. Izatt, James G. Fujimoto, Proc. of SPIE Vol. 6079

60792B, (2006) - 1605-7422/06/\$15 - doi: 10.1117/12.649024

Proc. of SPIE Vol. 6079 60792B-1 
tissue layer. It is difficult to determine the depth of the burn without biopsy; it requires a professional to make an educated guess after observing the injury for several days. The determination is usually made according to the texture and color of the burn and the size of the area affected by the burn. Evidently, a non-invasive imaging modality for rapidly evaluating burn depths would be of great help to physicians and patients. A potential technique is the polarization-sensitive optical coherence tomography (PS-OCT), ${ }^{4,5}$ which uses changes in polarization properties of thermally damaged tissue to assess the extent of burn injury. The birefringence seems to be the parameter that reveals the most information about the extent of the thermal damage.

As a branch of PS-OCT, Mueller-matrix OCT ${ }^{6}$ can provide complete characterization of the polarization properties of a sample by measuring the depth-resolved Jones matrix. A system with a single light source and continuous modulation of polarization states was previously reported by our group. ${ }^{7,8}$ This time, we report on the use of an improved high-speed, fiber-based Mueller-matrix OCT system with continuous source-polarization modulation for burn depth evaluation. The new system is capable of imaging at near video-quality frame rates $(8$ frames per second) and it has an improved depth penetration and resolution compared to the previous system. The applicability of the system for burn depth determination was demonstrated using biological samples of porcine tendon and porcine skin.

\section{EXPERIMENTAL SETUP}

The high-speed, continuously modulated, polarization sensitive Mueller-matrix OCT system is shown in Fig. 1. The light from the super-luminescent diode (Superlum; $\lambda_{0}=1.3 \mu \mathrm{m}, \mathrm{FWHM}-\mathrm{BW}=60 \mathrm{~nm}$, output power $10 \mathrm{~mW}$ ) is vertically polarized by a linear polarizer LPV. A polarization modulator (Conoptics; fast axis at $45^{\circ}$ ) continuously modulates source-polarization state with $140 \mathrm{kHz}$ sinusoidal waveform to ensure that multiple cycles of polarization states are applied to each segment of a depth scan. A linear polarizer oriented at $-45^{\circ}$ (LP -45) is placed in the reference arm to ensure equal intensities of reference light in both orthogonal polarization states.

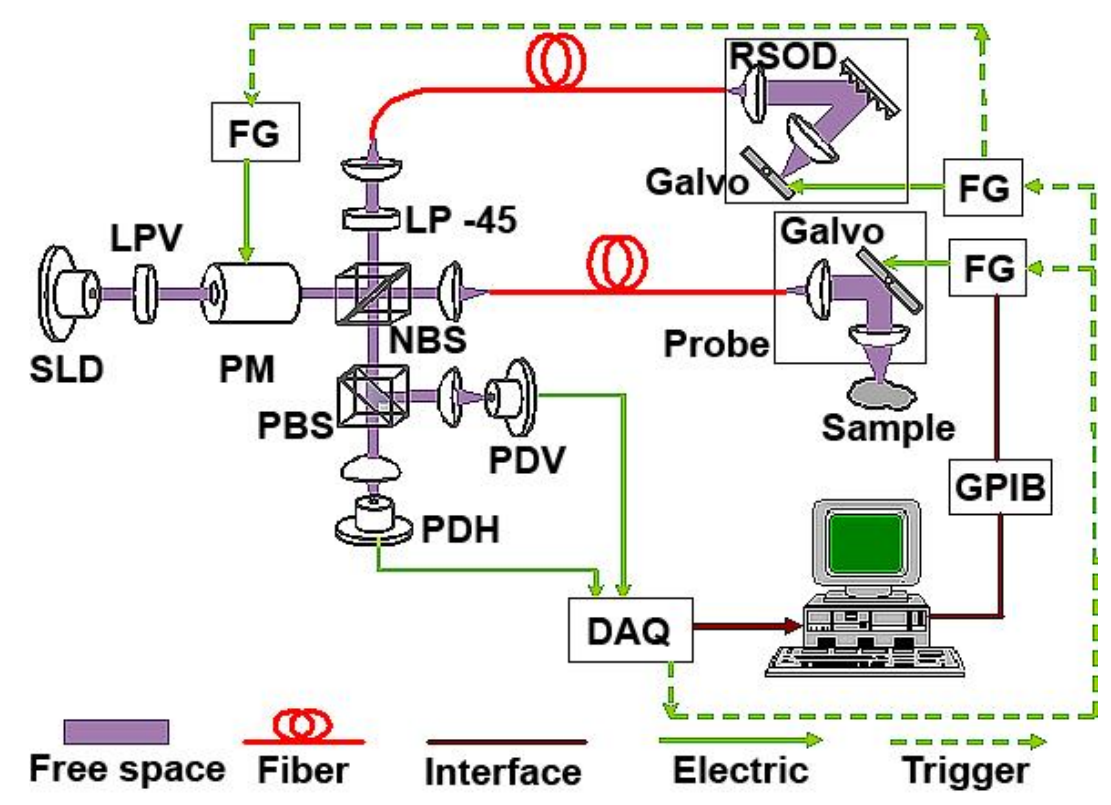

Fig. 1. Schematic of the Mueller-matrix PS-OCT system. SLD: superluminescent diode; PM: polarization modulator; NBS: non-polarizing beam splitter; PBS: polarizing beam splitter; PDH and PDV: photodiodes for $\mathrm{H}$ and V polarization; RSOD: rapid scanning optical delay line; LPV: vertical linear polarizer; LP $-45:-45^{\circ}$ linear polarizer; DAQ: data acquisition board; GPIB: GPIB interface; FG: function generator. 
The reference arm and the sample arm incorporate single mode optical fibers. A collimating lens, B-scan mirror and a focusing lens in the sample arm are integrated in a hand-held probe, which makes the in vivo experiments convenient. Two BK7 prisms are used in the sample arm for dispersion compensation and to adjust the height of the beam. A grating-based rapid scanning optical delay (RSOD) ${ }^{9}$ line is used for longitudinal scanning. The combined sample and reference light is split into horizontal and vertical polarization components by a polarizing beam splitter. The two orthogonal polarization components of the interference signal are detected by two photodiodes. The details of the algorithm for extracting the round-trip Jones matrix from the orthogonal intensity measurements are given in our previous paper. ${ }^{8}$ Slightly modified formulae including necessary modifications caused by the improvements in configuration of the sample and the reference arms were used here.

\section{RESULTS}

First, we verified the system's performance by measuring resolution and sensitivity. The resolution, which depends on the characteristics of the light source, was measured at $14 \mu \mathrm{m}$ in air $(10 \mu \mathrm{m}$ in biological tissue assuming the index of refraction of 1.4), which is comparable with the theoretical value of $12.5 \mu \mathrm{m}$. Sensitivity was measured to be $78 \mathrm{~dB}$.

Next, we proceeded to evaluate burn depths in porcine tendon and porcine ear skin ex vivo. The sample preparation included bathing the tendon in saline solution and scrubbing skin samples with glycerin after the hair was removed with hair removing lotion. For each sample, the measured Jones matrix at the sample surface was used to eliminate the polarization effect of the sampling fiber.

In the case of porcine tendon, a burn was induced on the side surface over the period of 20 seconds with the thermal cautery unit (Geiger Medical Tech.) heated to $90^{\circ} \mathrm{C}$. The intensity (a) and phase retardation (b) images of the thermally damaged porcine tendon are shown in Fig. 2. A typical banded structure seen in retardation image is clearly observable up to the depth of $1 \mathrm{~mm}$, which is an improvement of approximately $0.3 \mathrm{~mm}$ over the previously reported results. ${ }^{5,7}$ It results from the birefringence in porcine tendon and is the effect of 180 degree phase wrapping in phase retardation. While the intensity image does not show visible structural differences between intact (left of the white stripe) and burnt (right of the white stripe) areas, the phase retardation image exhibits clear disappearance of banded structure in the area marked with the dotted line. Since the type I collagen fibers are the predominant structure influencing the birefringence of tendons, the loss of birefringence can be attributed to the denaturation of collagen due to the exposure to high temperatures. The system is obviously capable of clearly localizing the burn location since the boundary of the affected region is well defined.

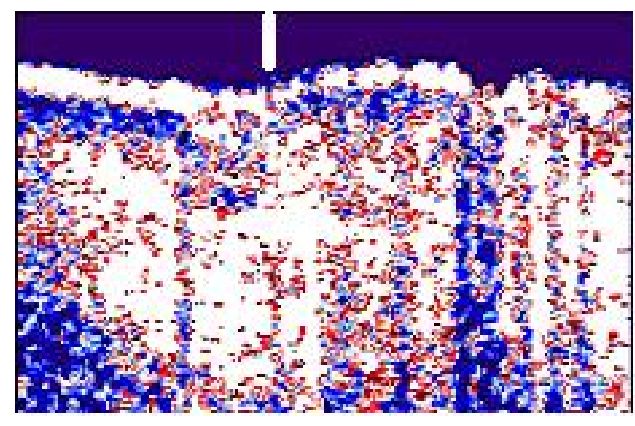

a.

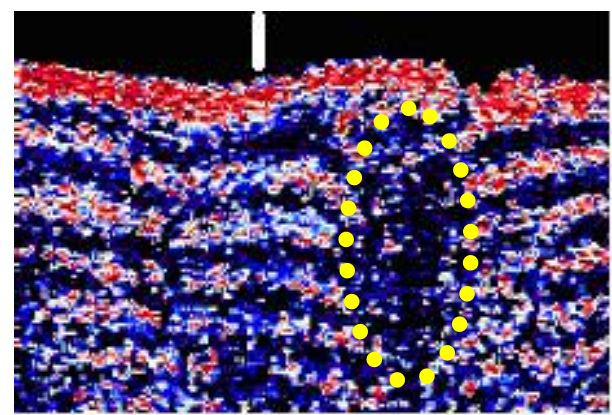

b.

Fig. 2. Intensity (a) and phase retardation (b) images of the thermally damaged porcine tendon. Dimensions of each image are $4 \mathrm{~mm} \times 1.2 \mathrm{~mm}$ (width $\mathrm{x}$ height).

Application of the system in burn depth determination was further investigated by imaging the porcine ear skin burnt for 20 seconds with the cauter heated to $150^{\circ} \mathrm{C}$. Upon completion of the OCT imaging, the skin tissue was fixed in $10 \%$ buffered neutral formalin and the paraffin-embedded tissue was sectioned and stained with 
hematoxylin and eosin (HE). For polarization microscopy unstained histological sections were deparaffinized and cover slip with water mount media. The stained sections were examined under an Olympus light microscope with 4X magnification to examine the thermally induced changes. Fig. 3 shows the OCT intensity (a) and phase retardation (b) images of a porcine skin sample after burning. Also, HE stained (c) and polarization (d) histology pictures are included for comparison. Again, the loss of birefringence is visible in the affected region marked with a line in Fig. 3. (b), (c), and (d) and can be attributed to the destruction of collagen-based connective tissue in the dermis. Although there are some changes in the intensity image as well, burn depth cannot be determined from this image. Comparison of phase retardation image with histology shows a good agreement in determining the burn depth. The ratio of burn's width and depth as determined from the retardation image is 2.60 and agrees well with the same ratio obtained from the histology, which is 2.67 . The difference in absolute lengths can be attributed to tissue shrinkage due to formalin fixation and the fact that the histology cut was potentially performed at a position slightly different from the one imaged with the OCT system. Based on the depth of the burn it can be classified as a fullthickness burn. Epidermis depth in the healthy region is also indicated in retardation $(0.16 \mathrm{~mm})$ and HE image $(0.13$ $\mathrm{mm}$ ) and shows a smaller difference between the two, which can be solely attributed to the post-fixation shrinkage.

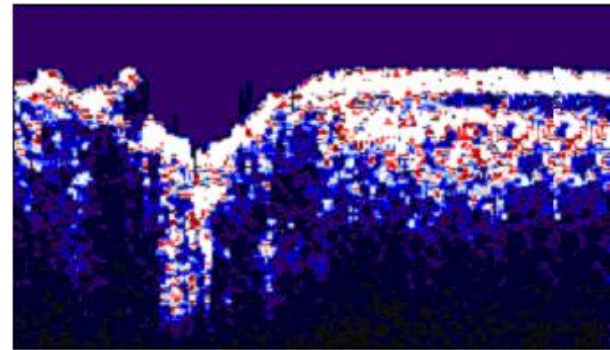

a.

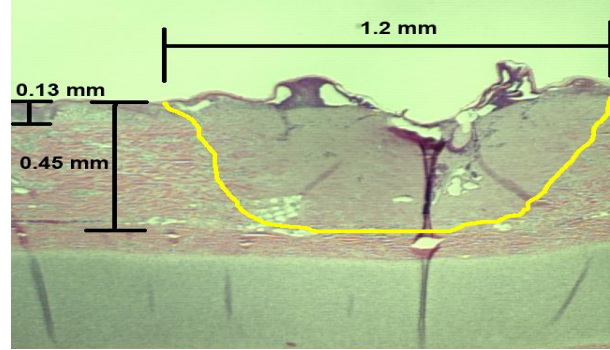

c.

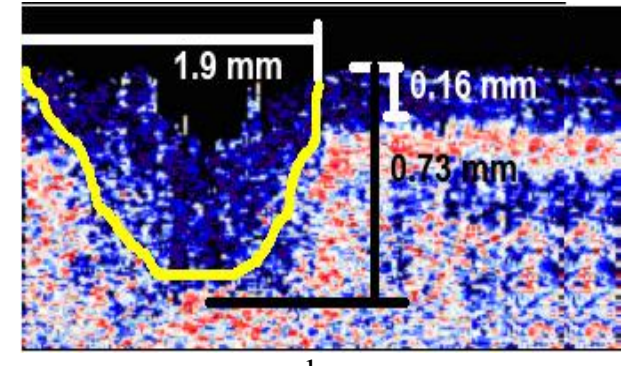

b.

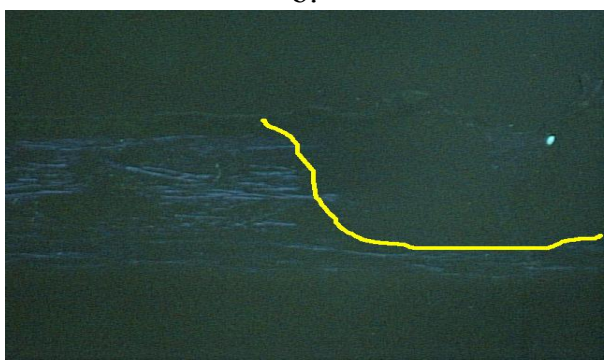

d.

Fig. 3. Intensity (a), phase retardation (b), HE stained histology (c) and polarization histology (d) images of a burnt porcine ear skin. Dimensions of images (a) and (b) are $4 \mathrm{~mm}$ x $1.2 \mathrm{~mm}$ (width $\mathrm{x}$ height).

\section{CONCLUSIONS}

In summary, we presented a high-speed, fiber-based Mueller-matrix OCT system with continuous sourcepolarization modulation and showed its applicability for burn depth determination. The system has an improved depth penetration, which was demonstrated in porcine tendon images. It is capable of accurately localizing changes in polarization properties due to thermal damage of tissue and, hence, has a potential to aid physicians in determining the extent of burn injuries.

A further extension of this work will include in vivo imaging of porcine skin, as well as the monitoring of the healing process over an extended period of time. The ability of the system to show the changes in optical polarization properties of tissues can lead to an efficient technique to instantaneously observe the post-burning recovery. 


\section{ACKNOWLEDGMENTS}

This project was sponsored by the Department of the Army (Cooperative Agreement Number: DAMD17-97-27016). The content of the information presented in this paper does not necessarily reflect the position or the policy of the government or NMTB. No official endorsement should be inferred.

\section{REFERENCES}

1. Burn Incidence and Treatment in the US: 2000 Fact Sheet, American Burn Association, (2000). [http://www.ameriburn.org/pub/BurnIncidenceFactSheet.htm]

2. R.S. Cotran, V. Kumar, and S.L. Robbins, Pathologic bases of disease $6^{\text {th }}$ edition, W.B. Saunders Co., Philadelphia, PA, 1999.

3. R. M. Johnson and R. Richards, "Partial-thickness burns: identification and management," Advances in skin \& wound care 16, No. 4, 178-187, 2003.

4. M. R. Hee, D. Huang, E. A. Swanson, and J. G. Fujimoto, "Polarization-sensitive low-coherence reflectometer for birefringence characterization and ranging," J. Opt. Soc. Am. B 9, 903-908, 1992.

5. J. F. de Boer, T. E. Milner, M. J. C. van Gemert, and J. S. Nelson, "Two-dimensional birefringence imaging in biological tissue by polarization-sensitive optical coherence tomography," Opt. Lett. 22, 934-936, 1997.

6. G. Yao and L.V. Wang, "Two-dimensional depth-resolved Mueller matrix characterization of biological tissue by optical coherence tomography," Opt. Lett. 24, 537-539, 1999.

7. M. Todorović, S. Jiao, G. Stoica, and L. Wang, "Determination of local polarization properties of biological samples in the presence of diattenuation by use of Mueller optical coherence tomography," Opt. Lett. 29, 24022404, 2004.

8. S. Jiao, M. Todorović, G. Stoica, and L. Wang, "Fiber-based polarization-sensitive Mueller-matrix optical coherence tomography with continuous source-polarization modulation," Appl. Opt. 44, 5463-5467, 2005.

9. G. J. Tearney, B. E. Bouma, and J. G. Fujimoto, "High-speed phase- and group-delay scanning with a gratingbased phase control delay line," Opt. Lett. 22, 1811-1813, 1997. 